\title{
Distinct S-wave reflectors (bright spots) detected beneath the Nagamachi-Rifu fault, NE Japan
}

\author{
Norihito Umino, Hisako Ujikawa, Shuichiro Hori, and Akira Hasegawa \\ Research Center for Prediction of Earthquakes and Volcanic Eruptions, Graduate School of Science, Tohoku University, Sendai 980-8578, Japan
}

(Received January 8, 2002; Revised September 20, 2002; Accepted September 26, 2002)

\begin{abstract}
Distinct phases reflected from mid-crustal reflectors ( $S x S$ and PxP phases) were observed in seismograms of aftershocks of 1998 M5.0 Sendai earthquake at nearby stations. We estimated the locations of the reflectors (bright spots) by using arrival time differences between these phases and direct waves. A clear reflector is located in the depth range of 15 to $21 \mathrm{~km}$ just beneath the fault plane of the M5.0 event. It dips toward the NNE direction with a dip angle of about $25^{\circ}$. Other reflectors are also located beneath the fault plane of the event. Internal structure of the $S$-wave reflector was estimated from spectral amplitude ratios of reflected $S x S$-wave to direct $S$-wave. Observed spectral ratios show that $S$-wave velocity in the reflector body is $\sim 1.1 \mathrm{~km} / \mathrm{s}$, and its thickness is about $50 \mathrm{~m}$. This suggests that a thin reflector body (bright spot) partially filled with fluids exists in the lower crust beneath the focal area of the 1998 event.
\end{abstract}

\section{Introduction}

On 15 September 1998, an earthquake with magnitude 5.0 occurred at $12 \mathrm{~km}$ depth beneath Sendai City, northeastern Japan, which caused slight damage in a portion of the city. The epicenter of this event was located about $10 \mathrm{~km}$ to the northwest of the Nagamachi-Rifu fault, an active fault zone running through the middle of the urban area of Sendai City in the NE-SW direction. Three days prior to the occurrence of the M5.0 earthquake, the first foreshock occurred and then active foreshock activity started at a location very close to the M5.0 main shock hypocenter. Waveforms of these foreshocks were similar to each other, suggesting that they occurred almost at the same locations with the same focal mechanisms (Umino et al., 2002).

Hori et al. (1999) revealed that distinct reflected $S x S$ and $P x P$ phases can be observed in seismograms of aftershocks of the M5.0 event at many stations surrounding its focal area. These reflected phases have similar features to those of the $S$ wave reflectors (bright reflective layers) extensively detected in the mid crust of the northeastern Japan arc (Hasegawa et al., 2000). We estimated the location of reflector beneath the focal area of the M5.0 earthquake from observed travel time differences between direct waves and reflected waves, and its internal structure from spectral amplitude ratio of reflected waves to direct waves.

\section{Locations of Reflectors}

Distinct reflected $P x P$ and $S x S$ phases were observed in seismograms of aftershocks of the M5.0 event at many nearby stations. Figure 1 shows locations of observation stations which recorded distinct $S x S$ and/or $P x P$ waves in seismograms of aftershocks. PxP phases and $S x S$ phases are

Copy right(c) The Society of Geomagnetism and Earth, Planetary and Space Sciences (SGEPSS); The Seismological Society of Japan; The Volcanological Society of Japan; The Geodetic Society of Japan; The Japanese Society for Planetary Sciences. observed at 4 stations and 20 stations, respectively. Aftershocks are distributed along the fault plane of the M5.0 event with a dimension of $3 \mathrm{~km} \times 3 \mathrm{~km}$. Figure 2 shows examples of PxP and $S x S$ phases recorded at IMZ station just above the M5.0 earthquake hypocenter. Predominant PxP phase is observed at vertical component of $1 \mathrm{~Hz}$ seismograph. Several $S x S$ phases are also recorded especially at SH component of $1 \mathrm{~Hz}$ seismograph. These $P x P$ and $S x S$ phases are shown by triangles and circles in Fig. 2, respectively.

The location of the most predominant reflector is estimated by inverting observed $P x P-P$ times at one station and $S x S-S$ times at 8 stations. In this estimation, the surface of the reflector is assumed to be a single plane (Horiuchi et al., 1988; Inamori et al., 1992). Hypocenters of the events used for this are determined precisely by the homogeneous station method (Ansell and Smith, 1975). The planar $S$-wave reflector is located at depths ranging from 15 to $22 \mathrm{~km}$. It spreads in an area of $\sim 10 \mathrm{~km} \times 10 \mathrm{~km}$ dipping to the northnortheast direction at an angle of $\sim 25^{\circ}$ (Fig. 3(a)). Standard deviations of the depths of the planar $S$-wave reflector are shown by dashed curves. Circles and triangles denote reflection points on the surface of the planar reflector with positive and negative residuals of $P x P-P$ and $S x S-S$ times, respectively.

Many other reflectors are also located by the image station method of Horiuchi et al. (1988). In this estimation, the location of a reflector can be determined by travel time differences for three or more earthquakes observed at a station. Depth distribution of reflectors thus determined by $P x P-P$ and $S x S$-S times are shown in Fig. 3(b) by short dashed and solid lines, respectively. Most of reflectors are located in the region between the fault plane of the M5.0 event and the planar reflector already located. These reflectors may cause several distinct $S x S$ phases shown in Fig. 2.

Asano et al. (1999) carried out seismic array observations 


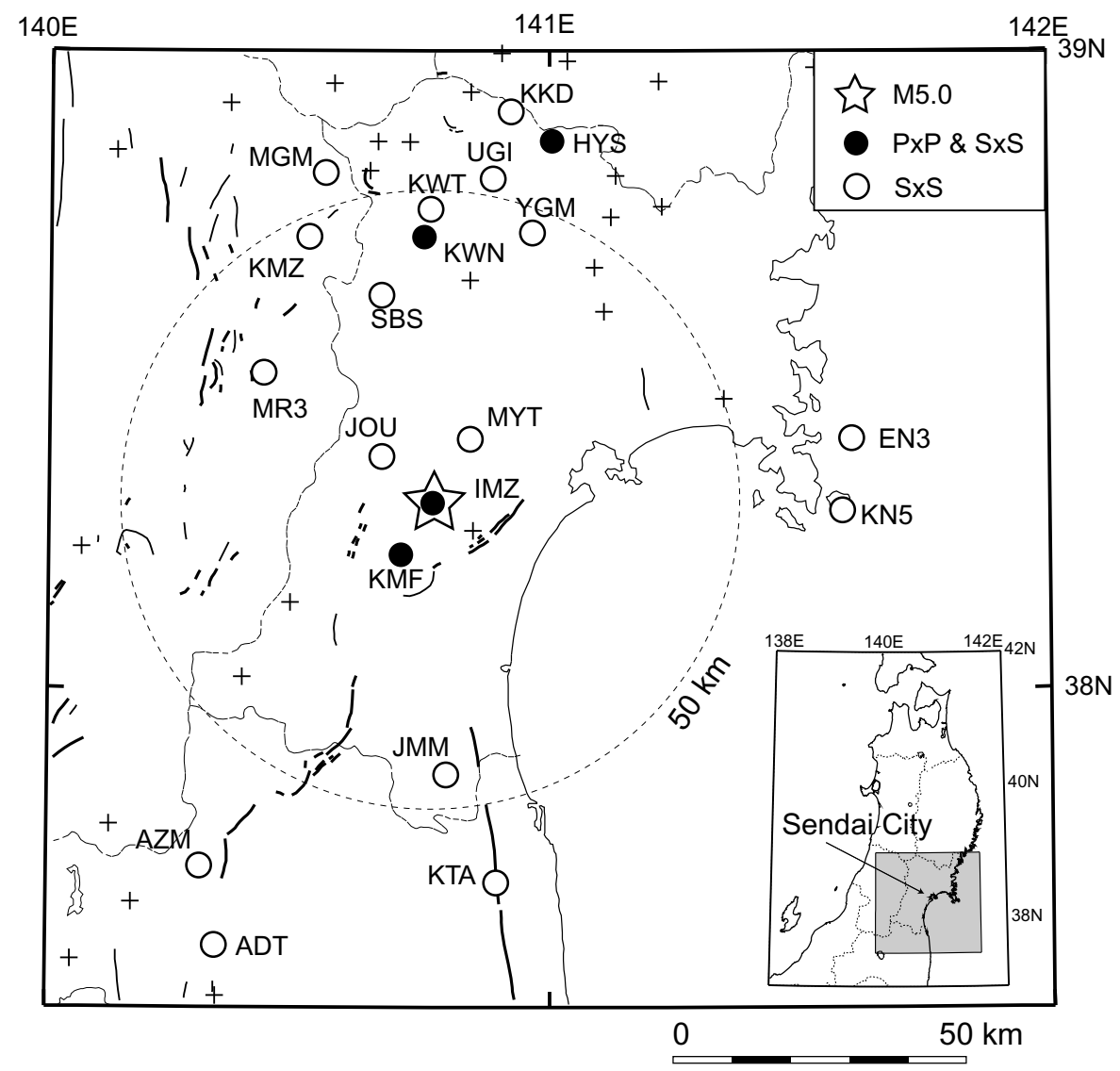

Fig. 1. Map showing observation stations and the epicenter of 1998 M5.0 Sendai earthquake. Open and solid circles denote the stations which recorded both $P x P$ and $S x S$ phases and only SxS phase, respectively. Crosses show the stations which recorded meither $P x P$ nor $S x S$ phase. Open star shows the epicenter of the M5.0 event.

in this area. They estimated spatial distribution of seismicwave scatterers by using observed array data. Estimated scatterers are located at almost the same locations as those of the $S$-wave reflectors presently estimated.

\section{Spectral Amplitude Ratio of Reflected $S x S$ - to Direct $S$-Waves}

In the present study, the internal structure of mid-crustal seismic-wave reflectors is estimated from spectral amplitude ratios of reflected $S x S$-waves to direct $S$-waves. Amplitude of direct $S$-wave from the $i$-th earthquake observed at $j$-th station at frequency $f \mathrm{~Hz}$ can be written as

$$
A_{i j}(f)=S_{i}(f) I_{j}(f, q) F_{i j} \exp \left(-2 p f t_{i j} Q-1\right) / r_{i j}
$$

where $S_{i}(f)$ is source spectrum of the $i$-th event, $F_{i j}$ is the factor of source radiation at $j$-th station, $I_{j}(f, q)$ is the station factor of $j$-th station including instrumental response, site amplification factor and free surface effect, $q$ is an incident angle to the surface, $t_{i j}$ and $r_{i j}$ denote travel time of direct wave and geometrical spreading factor, and $Q^{-1}$ is attenuation factor along the ray path. Amplitude of reflected $S x S$-wave with an incident angle $\alpha$ to the reflector at frequency $f \mathrm{~Hz}$ is expressed as

$$
\begin{aligned}
\operatorname{Aref}_{i j}(f)= & S_{i}(f) I_{j}\left(f, q^{\prime}\right) F_{i j}^{\prime} \\
& \times \exp \left(-2 p f t_{i j}^{\prime} Q-1\right) R(f, a) / r_{i j}^{\prime}
\end{aligned}
$$

where $R(f, a)$ is a reflection coefficient, $r_{i j}^{\prime}$ is the geometrical spreading factor of the reflected wave, $t_{i j}^{\prime}$ is travel time of the reflected wave from the $i$-th event to the $j$-th station. To remove the effect of the source factor, we take the amplitude ratio of $\operatorname{Aref}_{i j}(f)$ and $A_{i j}(f)$. Then

$$
\begin{aligned}
\left(\text { Aref }_{i j} f\right) / A_{i j}(f)= & I_{j}\left(f, q^{\prime}\right) F_{i j}^{\prime} r_{i j} \\
& \times \exp \left(-2 p f\left(t_{i j}^{\prime}-t_{i j}\right) Q-1\right) \\
& \times R(f, \alpha) /\left(I_{j}(f, q) F_{i j} r_{i j}^{\prime}\right) .
\end{aligned}
$$

We estimate amplitude ratios by using spectral amplitude ratio of reflected $S x S$-wave to direct $S$-wave.

\section{Estimation of the Internal Structure of $S$-Wave Reflector}

Since earthquakes used in this study is as small as magnitude $\sim 2$, individual focal mechanisms of these events could not be determined. Most of aftershocks whose mechanism solutions were determined have the same focal mechanism as the M5.0 main shock (Umino et al., 2002). So we used the focal mechanism of the M5.0 event to correct the effect of radiation patterns $\left(F_{i j}^{\prime} / F_{i j}\right)$ of these events. Travel times $\left(t_{i j}^{\prime}-t_{i j}\right)$ and geometrical spreading factors $\left(r_{i j} / r_{i j}^{\prime}\right)$ are calculated by assuming a homogeneous half space model with a constant $S$-wave velocity. To eliminate the effect of station factors $\left(I_{j}\left(f, q^{\prime}\right) / I_{j}(f, q)\right)$ from the amplitude ratio expressed by Eq. (3), we used only earthquakes for which 


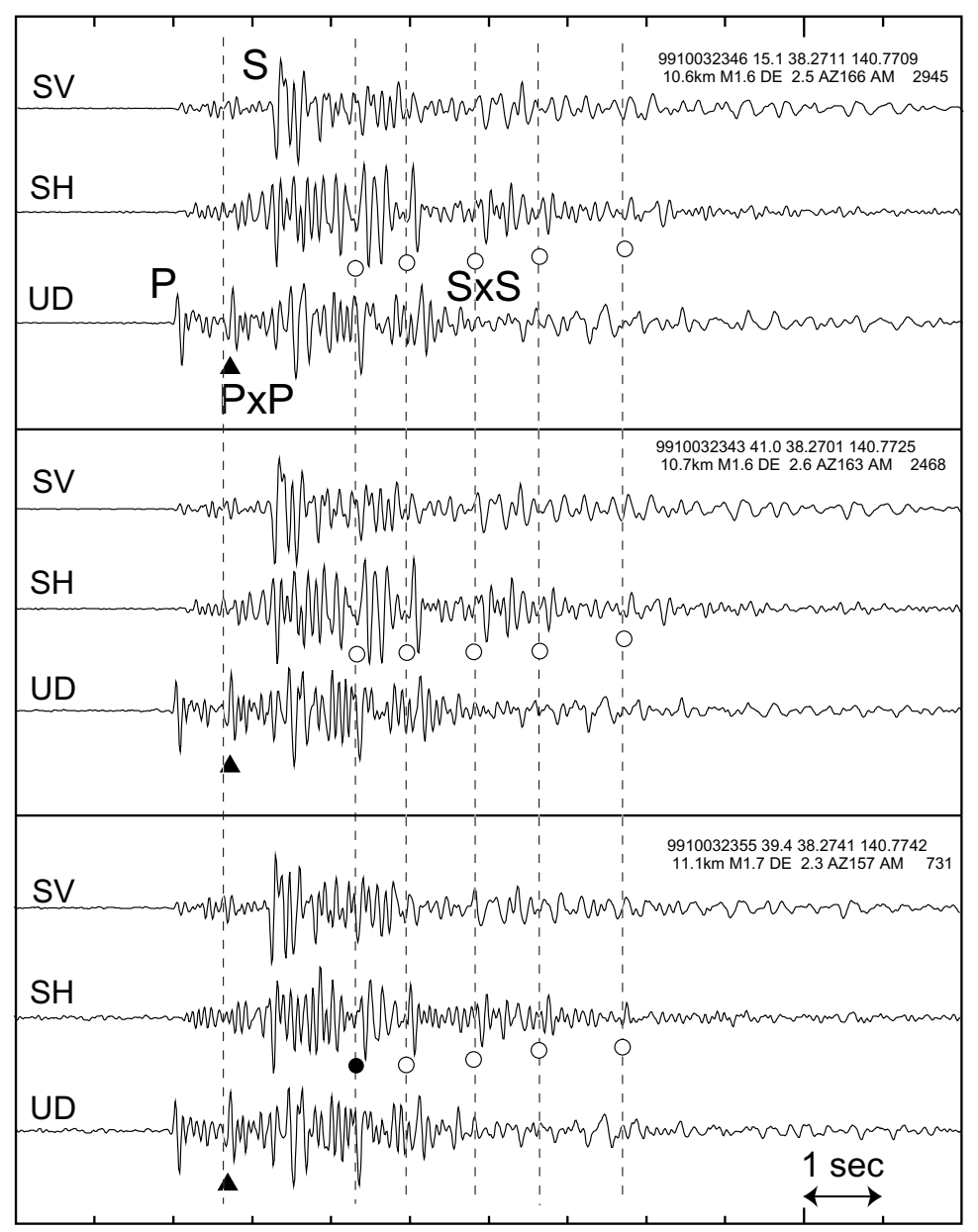

Fig. 2. Examples of seismograms for three earthquakes showing distinct later phases. Triangles and circles show $P x P$ and $S x S$ phases, respectively. Solid circles denote $S x S$ phases used in the spectral analysis shown in Fig. 5.

(a)

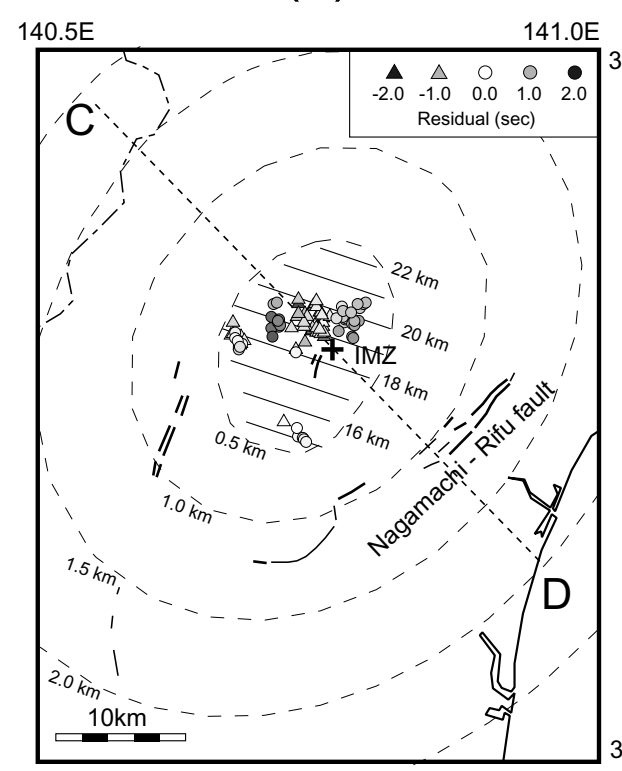

(b)

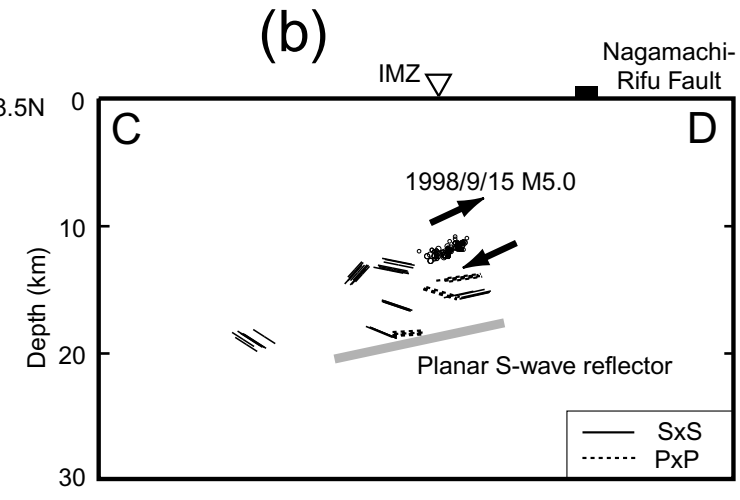

Fig. 3. Estimated planar $S$-wave reflectors beneath the focal area of the M5.0 earthquake. (a) Depth distribution of a planar $S$-wave reflector and reflection points on it. Depth to the planar reflector is shown by contours only in the area where the stabdard deviations of depths are smaller than $0.5 \mathrm{~km}$. Standard deviations of depth of the planar reflector are shown by dashed curves. Circles and triangles show reflection points with positive and negative residuals of $P x P-P$ and $S x S-S$ times, respectively. (b) Vertical cross section of the planar $S$-wave reflector and other reflectors along line CD in (a). Thick gray line shows the planar $S$-wave reflector. Thin solid and broken lines show other reflectors. Location of the Nagamachi-Rifu fault is shown by a thick line on the top. Thick arrows show the fault motion of the M5.0 main shock (Umino et al., 2002). 


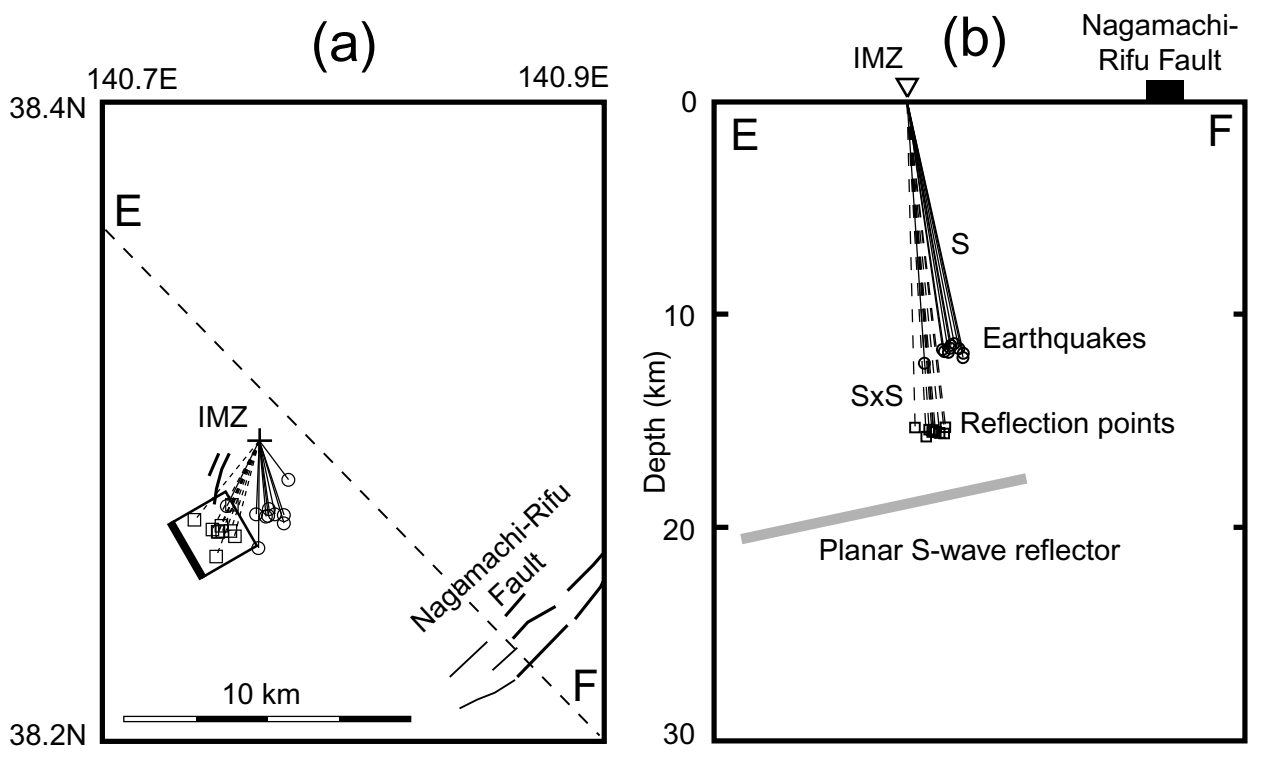

Fig. 4. Locations of hypocenters used (circles) and estimated reflection points (squares) in a spectral analysis. Seismic ray paths of direct $S$-waves and reflected $S x S$-waves are shown by solid and dashed lines, respectively. (a) Map view. The reflection plane is shown by a large square. Thick line of the square denotes the shallower segment of the reflection plane. The size of the square is arbitrary. (b) Vertical cross section along line EF in (a). The location of the planar $S$-wave reflector (see Fig. 3) is shown by thick gray line.

(a)

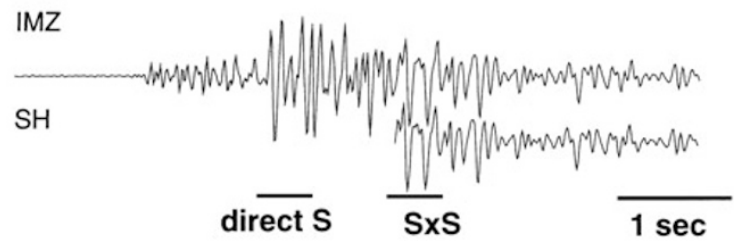

(b)

(c)
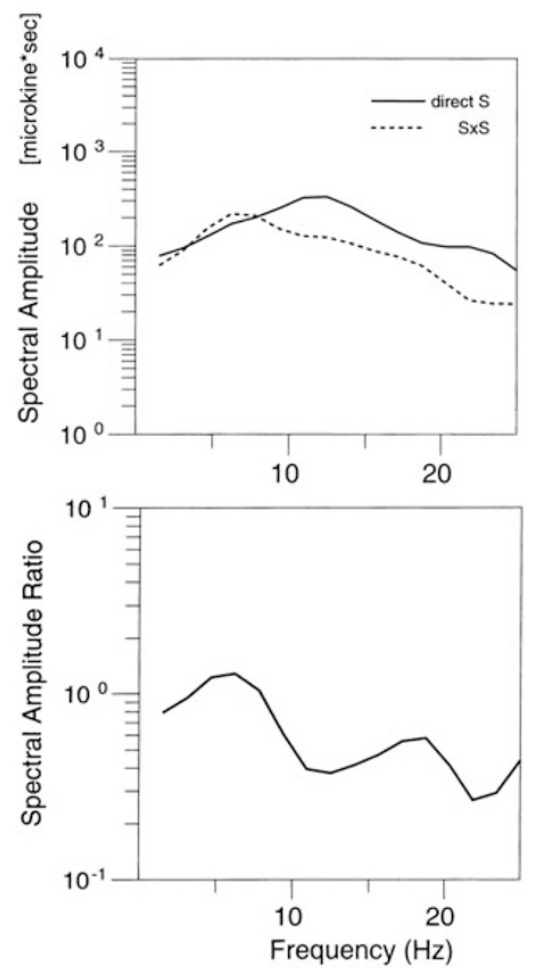

Fig. 5. An example of observed spectral amplitude ratio of $S x S$-wave to $S$-wave. (a) SH component seismogram for IMZ station (upper) and $\mathrm{SH}$ component seismogram for the surface of the reflector (lower). (b) Spectral amplitudes of direct $S$-wave (solid line) and $S x S$-wave (broken line). (c) Spectral amplitude ratio of $S x S$-wave to direct $S$-wave. (a)
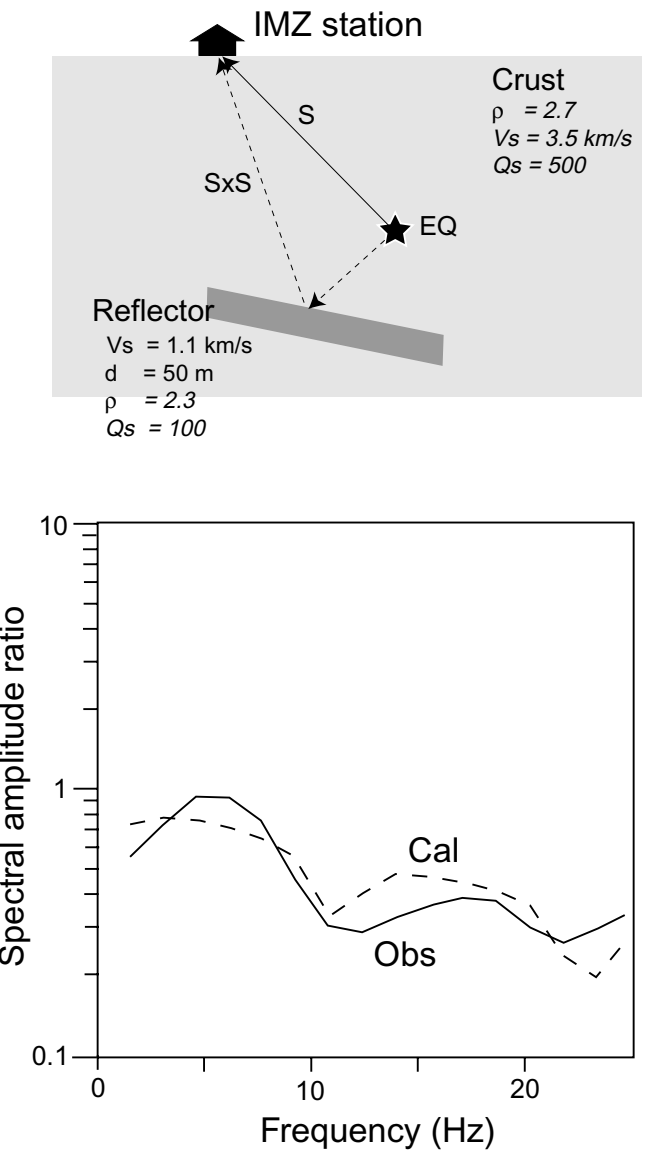

Fig. 6. (a) The most appropriate single-layer model of $S$-wave reflector. Densities and Qs values in the crust and the reflector are assumed in the grid search, and are shown by oblique letters. (b) Calculated spectral amplitude ratio from the most appropriate model (dashed line) and stacked spectral amplitude ratio (solid line). The stacked spectral amplitude ratio is corrected for the radiation pattern of focal mechanism and the geometrical spreading factor. 
(a)

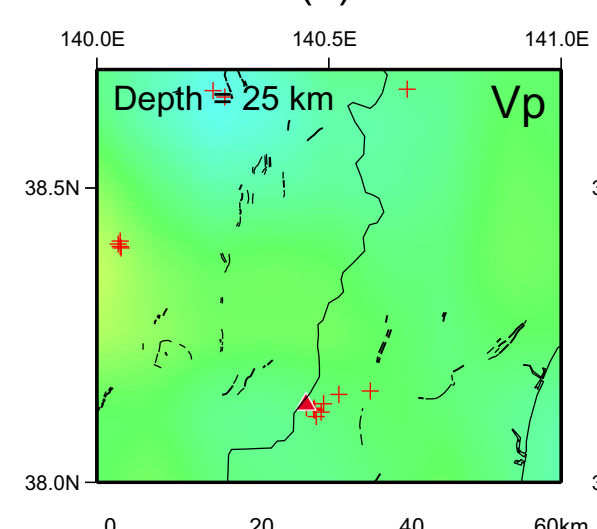

(b)

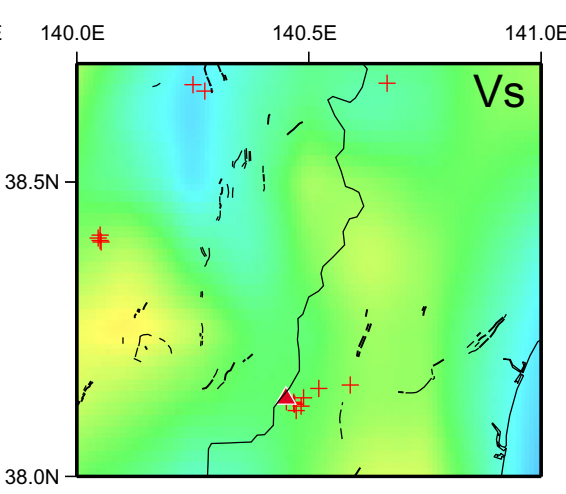

(c)

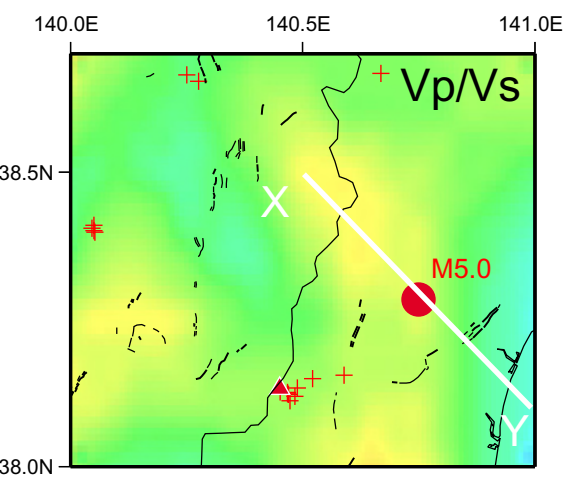

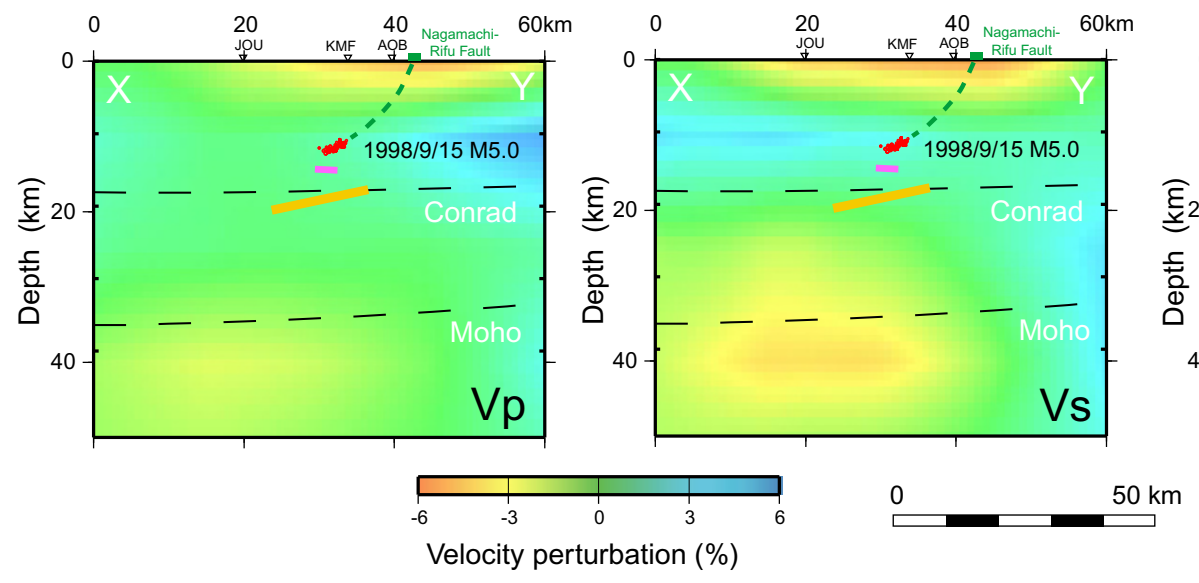

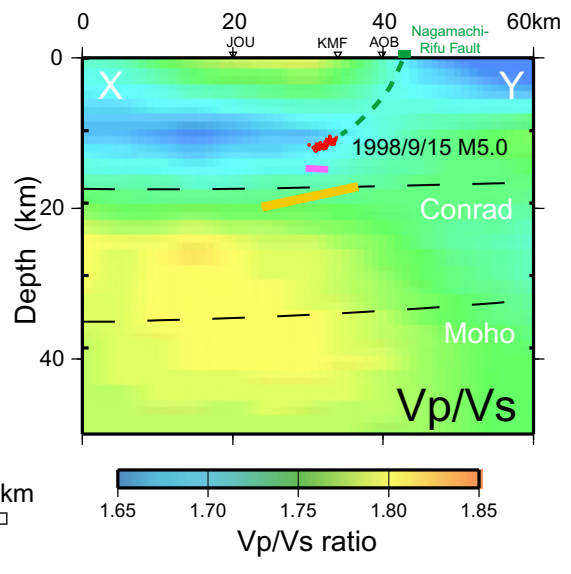

Fig. 7. Map views and vertical cross sections showing $S$-wave reflectors and seismic wave velocities. Distribution of $P$-wave velocity (a), $S$-wave velocity (b), and $\mathrm{Vp} / \mathrm{Vs}$ ratio (c) are shown by color scales, respectively (Nakajima et al., 2001). Orange and pink lines denote the planar $S$-wave reflector and the reflector whose thickness and $S$-wave velocity are investigated, respectively. Red circle and crosses show the epicenters of the M5.0 event and low-frequency microearthquakes (Okada and Hasegawa, 2000), respectively.

incident angles of reflected $S x S$-wave and direct $S$-wave are almost the same at a specific station. Assuming a station factor to be constant, frequency dependence on amplitude ratios of reflected $S x S$-wave to direct $S$-wave is proportional to that on reflection coefficient of the seismic-wave reflector $(R(f, \alpha))$.

Matsumoto and Hasegawa (1996) investigated the internal structure of an $S$-wave reflector beneath Nikko-Shirane volcano, northern Kanto District, central Japan, by using spectral amplitude ratios of reflected $S x S$-waves to direct $S$ waves. They revealed that the thickness of the $S$-wave reflector is of the order of $100 \mathrm{~m}$, and the reflector is probably filled with partially molten materials.

Based on their method of spectral amplitude ratios, we investigated the internal structure of the mid-crustal seismicwave reflector beneath the focal area of the M5.0 Sendai earthquake shown in Fig. 3(a). Distinct later phases are often observed on both vertical and SH components of seismograms from aftershocks of the M5.0 event. Figure 2 shows examples of the later phases recorded at IMZ station just above the main shock hypocenter.

As mentioned above, locations of reflection points of $S x S$ waves are determined by the image station method of Horiuchi et al. (1988). Reflection points for IMZ station are shown by open squares in Fig. 4. Ray paths of direct $S$-waves and those of reflected $S x S$-waves from reflection points are also shown in Fig. 4 by solid lines and dashed lines, respectively. Since the difference in incident angle between reflected $S x S$-wave and direct $S$-wave is estimated to be smaller than 5 degree at IMZ station, the site amplification factor is considered to be almost the same for reflected $S x S$ wave and direct $S$-wave.

Velocity amplitude spectra of reflected $S x S$-waves and direct $S$-waves are calculated from SH component and are shown in Fig. 5(b) by dashed and solid lines, respectively. Time windows of $0.5 \mathrm{~s}$ period for spectral analyses are shown by thick lines in Fig. 5(a). The spectra are smoothed by the hanning window technique. Spectral amplitude ratios of reflected $S x S$-wave to direct $S$-wave are estimated from these velocity amplitude spectra, and are also shown in Fig. 5(c).

As shown in Fig. 2, waveforms of earthquakes used are similar to each other and consequently the shapes of amplitude spectra are also similar to each other. We estimated spectral amplitude ratios for two similar events (M1.6 and M1.7) and stacked these spectral amplitude ratios. After correcting the effects of geometrical spreading factor and radiation pattern of focal mechanism, the stacked spectral amplitude ratio at IMZ station is shown by a solid line in Fig. 6(b). This observed spectral amplitude ratio shows two relatively large peaks with $\sim 11 \mathrm{~Hz}$ interval. We tried to find a reflector model, which can explain the frequency dependence of the observed spectral amplitude ratio, by using a grid search 
technique (Matsumoto and Hasegawa, 1996). The reflector body is assumed to be composed of one layer. We also assume that physical parameters, $P$ - and $S$-wave velocities and density, are constant both in the crust and reflector body. Densities and $Q$-values in the crust and the reflector body are assumed to be 2.7 and 500 (Umino and Hasegawa, 1984), 2.3 and 100, respectively. Moreover, assuming $S$-wave velocity in the crust to be $3.5 \mathrm{~km} / \mathrm{s}, S$-wave velocity in the reflector body and its thickness are estimated by the grid search technique.

The most appropriate model of the $S$-wave reflector is shown in Fig. 6(a) schematically. Theoretical spectral amplitude ratio for this model is shown by a broken line in Fig. 6(b). In this model, the $S$-wave velocity in the reflector body is $\sim 1.1 \mathrm{~km} / \mathrm{s}$, and its thickness is $\sim 50 \mathrm{~m}$. Since the length of time window for the spectral analyses of $S x S$ - and $S$-waves was taken as $0.5 \mathrm{~s}$ in this study, we cannot extract any information about the structure deeper than that corresponding to $0.5 \mathrm{~s}$ of propagation time. The maximum thickness corresponding to $0.5 \mathrm{~s}$ propagation time for $1.1 \mathrm{~km} / \mathrm{s}$ $S$-wave velocity is $\sim 550 \mathrm{~m}$, which is much thicker than the model presently obtained.

\section{Concluding Remarks}

Recently, precise 3D seismic wave velocity structure in the northeastern Japan arc was investigated by Nakajima et al. (2001). Figure 7 shows the map views and vertical cross sectional views of $P$-wave velocity $(V p), S$-wave velocity $(V s)$ and $V p / V s$ ratio obtained by them in this study area. A low $S$-wave velocity and large $V p / V s$ zone is located in the lower crust to the uppermost mantle just below the hanging wall side of the Nagamachi-Rifu fault. The planar $S$-wave reflector shown by a thick orange line and other reflectors not shown in this figure (see Fig. 3(b)) are located above this low $V p, V s$ and large $V p / V s$ zone. Nakajima et al. (2001) revealed that those low $V p, V s$ and large $V p / V s$ zones in the uppermost mantle beneath the northaestern Japan arc are caused by partial melting materials.

There is debate about the cause of $S$-wave reflectors and which of melting material and water is plausible for the internal material of the $S$-wave reflector body. Based on the spectral analysis of $S x S$-waves, the thickness and $S$-wave velocity of the reflector, which located just above the planar $S$-wave reflector, are estimated as to be $\sim 50 \mathrm{~m}$ and 1.1 $\mathrm{km} / \mathrm{sec}$. This close spatial relationship and such a thin low $S$-wave velocity layer (pink line in Fig. 7) as that presently estimated suggest that the $S$-wave reflectors are sill-like thin layers filled with $\mathrm{H}_{2} \mathrm{O}$, which may come from dehydration process in the low $V p, V s$ and high $V p / V s$ zone in the uppermost mantle. The $S$-wave reflectors (bright spots) are thought to play an important role in the modeling of deep slip processes in seismogenic inland fault systems. Several reflection events are observed in an extensive seismic experiment conducted in June 2001 around the Nagamachi-
Rifu fault (Nakamura et al., 2002). Some of those reflection events probably correspond to the $S$-wave reflectors obtained in this study. As shown in Fig. 2, distinct reflected PxPwaves are also observed at IMZ station. The reflection coefficient of the reflector body is large enough to reflect both $P$ - and $S$-waves simultaneously. Information about $P$-wave velocity in the reflector body, which constrains the internal structure of the reflector body, can be obtained from the same kind of analysis.

Acknowledgments. We would like to thank the members of Research Center for Prediction of Earthquakes and Volcanic Eruptions, Graduate School of Science, Tohoku University. Critical comments by H. Katao and S. Matsumoto helped to clarify this presentation.

\section{References}

Ansell, J. H. and E. G. C. Smith, Detailed structure of a mantle seismic zone using homogeneous station method, Nature, 253, 518-520, 1975.

Asano, Y., N. Umino, A. Nakamura, T. Okada, S. Hori, T. Kono, K. Nida, T. Sato, A. Hasegawa, M. Kosuga, and A. Hasemi, Spatial distribution of seismic scatterers beneath the Ou Backbone range, northeastern Japan, estimated by seismic array observations, J. Seism. Soc. Jpn., 52, 379394, 1999.

Hasegawa, A., A. Yamamoto, N. Umino, S. Miura, S. Horiuchi, D. Zhao, and H. Sato, Seismic activity and deformation process of the crust within the overriding plate in the northeastern Japan subduction zone, Tectonophys., 319, 225-239, 2000.

Hori, S., N. Umino, Y. Asano, and A. Hasegawa, S-wave reflectors in the crust of northeastern Japan, Programme and Abstracts The Seismological Society of Japan, 1999 Fall Meeting, p. 140, 1999.

Horiuchi, S., A. Hasegawa, A. Takagi, A. Ito, M. Suzuki, and H. Kameyama, Mapping of a melting zone near Mt. Nikko-Shirane in northern Kanto, Japan, as inferred from $S \times P$ and $S \times S$ reflections, Tohoku Geophys. J., 31, 43-55, 1988.

Inamori, T., S. Horiuchi, and A. Hasegawa, Location of mid-crustal reflectors by a reflection method using aftershock waveform data in the focal area of the 1984 Western Nagano Prefecture earthquake, J. Phys. Earth, 40, 379-393, 1992.

Matsumoto, S. and A. Hasegawa, Distinct S wave reflector in the midcrust beneath Nikko-Shirane volcano in the northeastern Japan arc, J. Geophys. Res., 101, 3067-3083, 1996.

Nakajima, J., T. Matsuzawa, A. Hasegawa, and D. Zhao, Three-dimensional structure of $V p, V s$ and $V p / V s$ beneath northeastern Japan: Implication for arc magmatism and fluids, J. Geophys. Res., 106, 21843-21857, 2001. Nakamura, A., Y. Asano, and A. Hasegawa, Estimation of deep fault geometry of the Nagamachi-Rifu fault from seismic array observations, Earth Planets Space, 54, this issue, 1027-1031, 2002.

Okada, T. and A. Hasegawa, Activity of deep low-frequency microearthquakes and their moment tensor in northeastern Japan, Bull. Volcanol. Soc. Jpn., 45, 47-63, 2000 (in Japanese with English abstract).

Umino, N. and A. Hasegawa, Three-dimensional Qs structure in the northeastern Japan arc, Bull. Volcanol. Soc. Jpn., 37, 217-228, 1984 (in Japanese with English abstract).

Umino, N., T. Okada, and A. Hasegawa, Foreshock and aftershock sequence of 1998 M5.0 Sendai, northeastern Japan, earthquake and its implications for earthquake nucleation, Bull. Seism. Soc. Am., 2002 (in press).

N. Umino (e-mail: umino@aob.geophys.tohoku.ac.jp), H. Ujikawa (e-mail: ujikawa@aob.geophys.tohoku.ac.jp), S. Hori (e-mail: hori@aob. geophys.tohoku.ac.jp), and A. Hasegawa (e-mail: hasegawa@aob.geophys. tohoku.ac.jp) 